Doug Geisler, Eva K. Grebel, and Dante Minniti, eds.

\title{
Massive Stars Ejected from R136?
}

\author{
Bernhard R. Brandl, David F. Chernoff \\ Center for Radio Astronomy and Space Research, Cornell University, \\ Ithaca, $N Y 14853, U S A$ \\ Anthony F. J. Moffat \\ Départment de physique, Université de Montréal, Montréal, Canada
}

\begin{abstract}
We present the observational signature of a statistically significant number of very massive stars around the core of R136 and discuss the evidence for dynamical processes to be responsible for their apparent location. Alternative scenarios are discussed as well.
\end{abstract}

\section{Observational Signatures}

The 30 Doradus region in the LMC (distance $\sim 52.5 \mathrm{kpc}$ ) is the largest and most massive H II region in the Local Group. Figure 1 shows the central $35^{\prime \prime} \times 35^{\prime \prime}$. Note the location of the labeled emission line stars, the most massive stars in that region. The ring-like constellation of these stars around the cluster center is obvious! About one third of the emission line stars in 30 Dor are located within $4^{\prime \prime}$ of R136a, close to another third is spread across the entire region, with distances ranging from $16^{\prime \prime} \ldots 135^{\prime \prime}$. But more than one third of these massive stars in 30 Dor appear to be located in a shallow sphere of $r \approx 8^{\prime \prime}-13^{\prime \prime}$ (although even more of the massive stars, which we attribute to the core region, may be actually located in the sphere behind or in front of R136a). Could this be just a statistical fluctuation? For a core radius of $r_{c} \approx 1^{\prime \prime}$ (Brandl et al. 1996) and under the assumption that the stellar density distribution follows roughly a standard cluster King profile, we would expect only two stars, while we find 9 well above a random statistical fluctuation of \pm 3 stars! Thus we conclude that their apparent location must be due to some physical process.

\section{Possible Scenarios}

The detailed mathematical treatment of the ejection mechanism will be the subject of a subsequent paper. Here we just state that two boundary conditions may lead to an enhanced interaction rate between massive stars: the high binary frequency amongst massive stars (Preibisch et al. 2000), and mass segregation (Portegies-Zwart et al. 1999) or initial subclustering, leading to an overdensity of massive stars in the center. The latter one has been observed by Brandl et al. (1996) and is shown in Figure 2a. For an ejection velocity of $15 \mathrm{~km} / \mathrm{s}$ and 

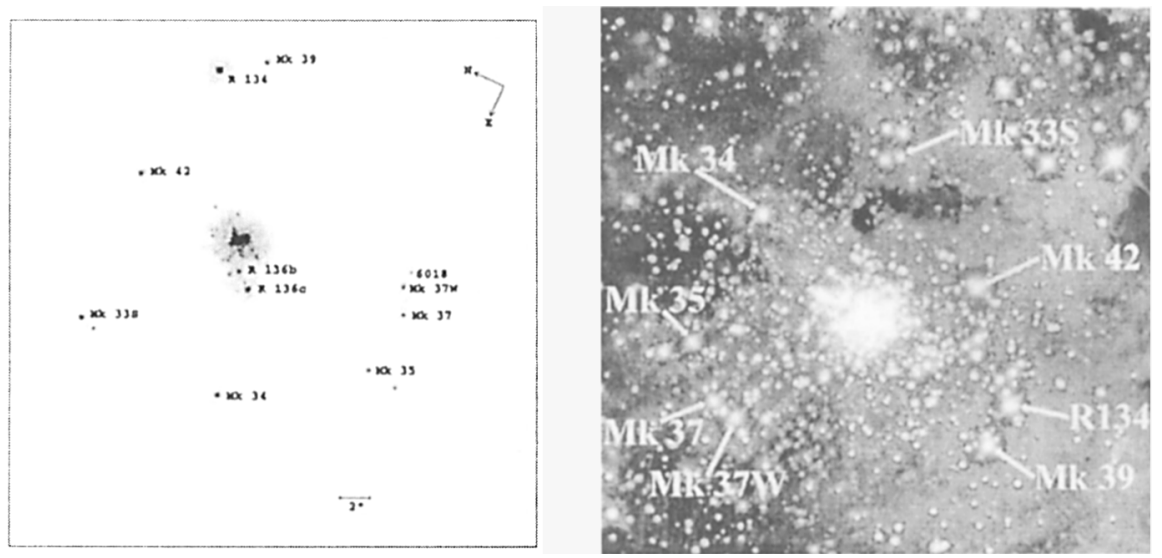

Figure 1. Left: PC6 F469N [HeII] line image of R136 (Parker 1995). $\mathrm{R} 136 \mathrm{a}$ is the entire central cluster of stars. Emission line stars are labeled. Right: Deep multiband WFPC2 image (Maiz-Apellániz, Walborn \& Barba 2001).

an average projection factor of $\cos (45)$ the massive stars would have taken only 0.25 Myr to reach their apparent location.

Other explanations might include:

- Independent Sites of Local Star Formation: Massive stars form in clusters and associations together with less massive stars. According to a standard Salpeter inital mass function (Salpeter 1955) one would expect about 65 OB stars $(m \geq$ $4 \mathrm{M}_{\odot}$ ) for each star of $m \geq 60 \mathrm{M}_{\odot}$. We've analyzed Hunter et al.'s (1996) WFPC2 data in the V (F555W) and I (F814W) filter bands and derived the local luminosity functions within $0.5 \mathrm{pc}\left(2^{\prime \prime}\right)$ around 8 massive stars and 16 randomly chosen test fields The result is shown in Figure 2b: we find no significantly enhanced stellar density around the peculiar emission line stars that would support the scenario of localized star forming events, independent of R136.

- Sequential Star Formation: Sequential star formation has been found to have triggered the LMC associations LH9 and LH10 (Parker et al. 1992) as well as N144 and N158 (Lortet \& Testor 1988) in the vicinity of the 30 Dor nebula. Several sites of ongoing star formation, such as Hodge 301 and R143, are located only $3^{\prime}$ from R136. As Figure $1 \mathrm{~b}$ indicates, our peculiar emission line stars are located very close to R136, in a region where most of the gas has been cleared out (unlike the regions mentioned above). Furthermore, the emission line stars around R136 are on average at least the same age as the stars within R136a and do not show the age spread expected from triggered star formation.

- Supernova Explosions: Asymmetries in the ejected shell from a type II supernova produce a recoil which kicks the star away from its birthplace. Both components remain bound and form a runaway OB star with a neutron star or black hole companion. Although Mk 34 is probably a high-mass X-ray binary (Wang 1995), R136 is too young to have produced so many supernovae. 

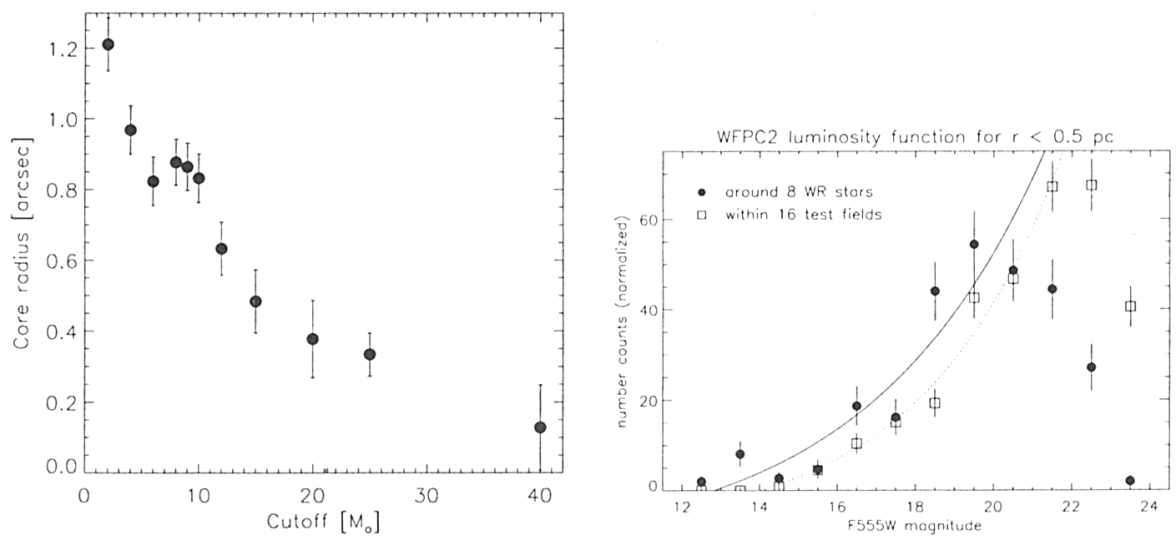

Figure 2. Left: The core radius of R136 as a function of the lower mass limit of stars taken into account; the more massive stars are clearly more concentrated toward the center (Brandl et al. 1996). Right: A comparison between the local luminosity functions within $\overline{0.5 \mathrm{pc}}\left(2^{\prime \prime}\right)$ for 16 random test fields $(8$ closer to the core and 8 farther than the "sphere") and near the 8 emission line stars in the sphere; the counts were normalized to the radial cluster density and averaged.

\section{Conclusions}

Based on theoretical expectation and the lack of good alternative scenarios we conclude that the peculiar concentration of the most massive stars $2-3 \mathrm{pc}$ around the core of R136a is probably the result of stellar interactions that have led to the ejection of the stars from their birthplace in R136a.

Acknowledgments. We'd like to thank Eva Grebel and Doug Geisler for the excellent organization of the first IAU Symposium in Chile.

\section{References}

Brandl, B. et al. 1996, ApJ, 466, 254

Hunter, D. A. et al. 1996, ApJ, 459, 27

Lortet, M.-C. \& Testor, G. 1988, A\&A, 178, 25

Maíz-Apellániz, J., Walborn, N. R. \& Barbá, R. 2002, these proceedings

Parker, J.W., Garmany, C.D., Massey, P., \& Walborn, N.R. 1992, AJ, 103, 1205

Parker, J. W., Heap, S. R. \& Malumuth, E. M. 1995, ApJ, 448, 705

Portegies Zwart, S. F., Makino, J., McMillan, S. L. W., Hut, P. 1999, A\&A, 348,117

Preibisch, Th. et al. 2000 in IAU Symp. 200, Birth and Evolution of Binary Stars, eds. B. Reipurth \& H. Zinnecker, (San Francisco: ASP), 106

Salpeter, E. E. 1955, ApJ, 121, 161

Wang, Q. D. 1995, ApJ, 453, 783 